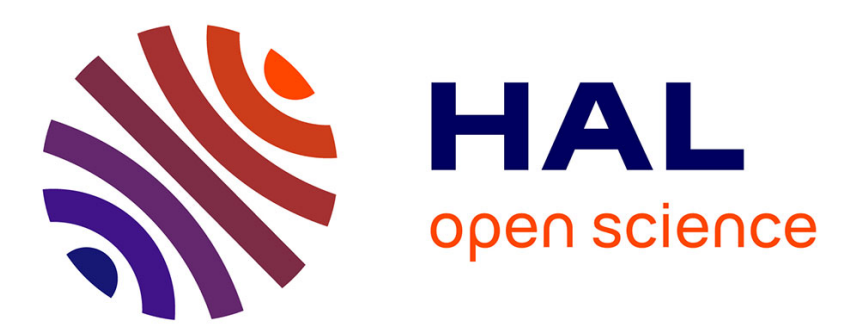

\title{
Density Conditions for Triangles in Multipartite Graphs
} Adrian Bondy, Jian Shen, Stéphan Thomassé, Carsten Thomassen

\section{To cite this version:}

Adrian Bondy, Jian Shen, Stéphan Thomassé, Carsten Thomassen. Density Conditions for Triangles in Multipartite Graphs. Combinatorica, 2006, 26, pp.121-131. 10.1007/s00493-006-0009-y . lirmm00140328

\section{HAL Id: lirmm-00140328 https://hal-lirmm.ccsd.cnrs.fr/lirmm-00140328}

Submitted on 11 Jun 2007

HAL is a multi-disciplinary open access archive for the deposit and dissemination of scientific research documents, whether they are published or not. The documents may come from teaching and research institutions in France or abroad, or from public or private research centers.
L'archive ouverte pluridisciplinaire HAL, est destinée au dépôt et à la diffusion de documents scientifiques de niveau recherche, publiés ou non, émanant des établissements d'enseignement et de recherche français ou étrangers, des laboratoires publics ou privés. 


\title{
Density conditions for triangles in multipartite graphs
}

\author{
Adrian Bondy, Jian Shen, Stéphan Thomassé, Carsten Thomassen
}

\begin{abstract}
We consider the problem of finding a large or dense triangle-free subgraph in a given graph $G$. In response to a question of P. Erdős, we prove that, if the minimum degree of $G$ is at least $17|V(G)| / 20$, the largest triangle-free subgraphs are precisely the largest bipartite subgraphs in $G$. We investigate in particular the case where $G$ is a complete multipartite graph. We prove that a finite tripartite graph with all edge densities greater than the golden ratio has a triangle and that this bound is best possible. Also we show that an infinite-partite graph with finite parts has a triangle, provided that the edge density between any two parts is greater than $1 / 2$.
\end{abstract}

\section{Introduction.}

Let $G$ be a $n$-partite graph on the finite sets $X_{1}, \ldots, X_{n}$. Suppose that every vertex $x$ of $G$ has a positive weight $w(x)$. The weight $w(S)$ of a subset $S$ of vertices is defined as the sum of the weights of the vertices in $S$. The weight of an edge $x y$ is defined as $w(x y):=w(x) w(y)$. The edge density of the restriction of $G$ to the bipartite subgraph of $G$ induced by $X_{i} \cup X_{j}$ is the sum of the weights of the edges between $X_{i}$ and $X_{j}$ divided by $w\left(X_{i}\right) w\left(X_{j}\right)$. The total density of $G$ is the sum of all edge densities. Clearly, a graph may be regarded as a weighted graph in which all weights are equal to 1 . All graphs are simple, that is, without multiple edges, and also finite, except in Theorem 2.

A transversal of $G$ is a subgraph of $G$ induced by a set $X$ such that $\left|X \cap X_{i}\right|=1$ for each $i$, $1 \leq i \leq n$.

Lemma 1 If $r$ is the total density of the multipartite graph $G$, some transversal of $G$ has at least $\lceil r\rceil$ edges.

Proof. The sum, taken over all the transversals $X$ of $G$, of $|E(X)|$ is equal to $r\left|X_{1}\right|\left|X_{2}\right| \ldots\left|X_{n}\right|$, since each edge between $X_{i}$ and $X_{j}$ is counted $\left|X_{1}\right|\left|X_{2}\right| \ldots\left|X_{n}\right| /\left(\left|X_{i}\right|\left|X_{j}\right|\right)$ times. Thus, some transversal has at least $\lceil r\rceil$ edges.

Let $H$ be a graph. Denote by $\chi(H)$ the chromatic number of $H$ and by ex $(H, n)$ the maximum number of edges in a graph on $n$ vertices containing no copy of $H$. For all $n \geq 1$, denote by 
$d_{n}(H)$ the minimum $d$ such that every $n$-partite graph in which all edge densities are strictly greater than $d$ contains a copy of $H$. The definition of $d_{n}(H)$ does not depend on the graph $G$ being weighted or unweighted, since every real number can be approximated by a rational number. Clearly $d_{n+1}(H) \leq d_{n}(G)$ for all $n \geq \chi(H)$, so $d(H):=\lim _{n \rightarrow \infty} d_{n}(H)$ exists.

Corollary 1 Let $G$ be an n-partite graph with total density strictly greater than $\operatorname{ex}(H, n)$. Then some transversal of $G$ contains a copy of $H$.

\section{Corollary 2}

$$
d_{n}(H) \leq \frac{\operatorname{ex}(H, n)}{\left(\begin{array}{l}
n \\
2
\end{array}\right)}
$$

\section{Theorem 1}

$$
d(H)=\frac{\chi(H)-2}{\chi(H)-1}
$$

Proof. By Corollary 2,

$$
d(H) \leq \lim _{n \rightarrow \infty} \frac{\operatorname{ex}(H, n)}{\left(\begin{array}{l}
n \\
2
\end{array}\right)} .
$$

But, by the Erdős-Stone-Simnovits theorem (see Erdős and Simonovits [2]),

$$
\lim _{n \rightarrow \infty} \frac{\operatorname{ex}(H, n)}{\left(\begin{array}{l}
n \\
2
\end{array}\right)}=\frac{\chi(H)-2}{\chi(H)-1} .
$$

Thus

$$
d(H) \leq \frac{\chi(H)-2}{\chi(H)-1}
$$

On the other hand, the $n$-partite graph $G$ obtained from the empty graph on a set $V$ of $n$ vertices by splitting each vertex $v$ of $V$ into $\chi(H)-1$ vertices $v_{1}, v_{2}, \ldots, v_{\chi(H)-1}$, and joining two vertices $x_{i}$ and $y_{j}$ if and only if $x \neq y$ and $i \neq j$, has all edge densities equal to $\frac{\chi(H)-2}{\chi(H)-1}$. Moreover, this graph does not contain $H$ because it is a $(\chi(H)-1)$-partite graph (with parts $\left.X_{i}:=\left\{v_{i}: v \in V\right\}, 1 \leq i \leq \chi(H)-1\right)$. This establishes the opposite inequality

$$
d(H) \geq \frac{\chi(H)-2}{\chi(H)-1}
$$

\section{Infinite-partite graphs.}

Define $t_{n}:=d_{n}\left(K_{3}\right)$, where $K_{3}$ is the triangle. By Theorem 1 , the limit of $t_{n}$, as $n$ tends to infinity, is $1 / 2$. However, this result does not imply the existence of $t_{\omega}$, where $t_{\omega}$ is the smallest real number such that every infinite-partite graph with finite parts and all edge densities strictly greater than $t_{\omega}$ has a triangle. One needs an additional argument. 
Lemma 2 Let $G$ be a 4-partite graph, one of whose parts is a singleton $\{x\}$. If the minimal edge density is greater than 1/2, G has a triangle.

Proof. Suppose that $G$ has no triangle. Let $A, B$ and $C$ be the parts distinct from $\{x\}$ and let $A_{1}, B_{1}$ and $C_{1}$ be the sets of neighbours of $x$ in $A, B$ and $C$, respectively. Set $A_{2}=A \backslash A_{1}$, $B_{2}=B \backslash B_{1}$ and $C_{2}=C \backslash C_{1}$. Note that there is no edge between $A_{1}, B_{1}$ and $C_{1}$; observe also that $\left|A_{2}\right|<\left|A_{1}\right|,\left|B_{2}\right|<\left|B_{1}\right|$ and $\left|C_{2}\right|<\left|C_{1}\right|$. Consider now the 4-partite subgraph induced by $A_{1}, A_{2}, B_{1}, B_{2}$. Its total density is greater than 2 . Indeed, if we fix the number of edges, the minimal total density is achieved by minimizing the number of edges between $A_{2}$ and $B_{2}$. Since $\left|A_{1}\right|\left|B_{2}\right|+\left|B_{1}\right|\left|A_{2}\right|<|A||B| / 2$, the total density must exceed 2. Thus, by Lemma 1 , there is a transversal with at least seven edges in the 6 -partite subgraph induced by $A_{1}, A_{2}, B_{1}, B_{2}, C_{1}, C_{2}$. Since the nine possible types of edges can be partitioned into the three tripartite subgraphs: $A_{1} B_{2} C_{2}, B_{1} C_{2} A_{2}$ and $C_{1} A_{2} B_{2}$, one of them yields a triangle in the transversal.

Theorem 2 t $t_{\omega}$ exists and $t_{\omega}=1 / 2$.

Proof. Let $G$ be an infinite-partite graph on finite classes with all edge densities greater than $1 / 2$. Let $X$ be any part of $G$. At least one vertex $x$ of $X$ is joined to more than one half of the vertices of infinitely many classes. Pick three of these classes $A, B$ and $C$, and apply lemma 2 to the subgraph of $G$ induced by $A, B, C,\{x\}$.

\section{Tripartite graphs and the golden ratio.}

Let $(G, w)$ be a weighted tripartite graph $G$ with parts $A, B$ and $C$. We denote the edge density between $A$ and $B$ by $\gamma$, the edge density between $B$ and $C$ by $\alpha$ and the edge density between $C$ and $A$ by $\beta$. We say that $\alpha, \beta, \gamma$ is a cyclic triple if the following conditions are satisfied:

$$
\alpha \beta+\gamma>1, \beta \gamma+\alpha>1 \text { and } \gamma \alpha+\beta>1
$$

If the edge densities of $G$ form a cyclic triple, then $G$ is said to be cyclic. To every triple which is not cyclic, for instance with $\beta \gamma+\alpha \leq 1$, we associate a weighted triangle-free graph on sets $A^{\prime}$, $B^{\prime}$ and $C^{\prime}$ with weight function $w^{\prime}$ in the following way: $A^{\prime}=\{a\}$ with $w^{\prime}(a)=1, B^{\prime}=\left\{b_{1}, b_{2}\right\}$ with $w^{\prime}\left(b_{1}\right)=\gamma$ and $w^{\prime}\left(b_{2}\right)=1-\gamma, C^{\prime}=\left\{c_{1}, c_{2}\right\}$ with $w^{\prime}\left(c_{1}\right)=\beta$ and $w^{\prime}\left(c_{2}\right)=1-\beta$, the edges of $G$ forming the 5 -circuit $a b_{1} c_{2} b_{2} c_{1} a$. Observe that the edge densities $\alpha^{\prime}, \beta^{\prime}, \gamma^{\prime}$ of this graph satisfy $\alpha^{\prime}=1-\beta \gamma \geq \alpha, \beta^{\prime}=\beta$, and $\gamma^{\prime}=\gamma$. The golden ratio, denoted by $\tau$, is the positive solution of the equation $x^{2}+x=1$. Note that $\alpha=\beta=\gamma=\tau$ is not a cyclic triple, and thus these densities can be realized by a triangle-free tripartite graph. This shows that $t_{3} \geq \tau$.

Theorem 3 If $G$ is cyclic, it contains a triangle.

Proof. Without loss of generality, we can assume that the total weights of $A, B$ and $C$ are each equal to 1 . Let $G$ be a weighted counterexample with as few vertices as possible. For each vertex $a_{i}$ of $A$, we let $\gamma_{i}$ (resp. $\beta_{i}$ ) denote the weight of the neighbourhood of $a_{i}$ in $B$ (resp. $C$ ). By definition, $\beta=\sum \omega\left(a_{i}\right) \beta_{i}$ and $\gamma=\sum \omega\left(a_{i}\right) \gamma_{i}$. Let $\left(\beta^{\prime}, \gamma^{\prime}\right)$ be an element of the boundary of the convex hull $\left\{\sum x_{i}\left(\beta_{i}, \gamma_{i}\right): \sum x_{i}=1\right.$ and $\left.x_{i} \geq 0\right\}$ which is in the positive cone pointed at 
$(\beta, \gamma)$. We then have $\beta^{\prime} \geq \beta$ and $\gamma^{\prime} \geq \gamma$. Moreover $\sum x_{i}\left(\beta_{i}, \gamma_{i}\right)=\left(\beta^{\prime}, \gamma^{\prime}\right)$ in such a way that only two values $x_{i}$ are non-zero. Thus, since $G$ is minimal, we may suppose that $|A| \leq 2$, because the vertices $a_{i}$ of $A$ for which $x_{i}=0$ can be deleted and the weight of the remaining vertices can be modified so that the resulting graph is a counterexample to Theorem 3. Furthermore, in the case that $|A|=2$, say $A=\left\{a_{1}, a_{2}\right\}$, we may suppose that

$$
\text { either } \beta_{1}<\beta_{2} \text { and } \gamma_{1}>\gamma_{2} \text { or } \beta_{1}>\beta_{2} \text { and } \gamma_{1}<\gamma_{2} \text {, }
$$

for otherwise we could have transferred the weight of one vertex of $A$ to the other vertex of $A$ to get a counterexample with fewer vertices. The same condition holds for $B$ and $C$. If one of the classes has only one vertex, we immediately conclude that $G$ has a triangle since it is cyclic. So $|A|=|B|=|C|=2$.

Suppose first that every vertex has at least one neighbour in each of the other classes. Then each vertex must have exactly one neighbour in each of the other classes. Let $A=\left\{a_{1}, a_{2}\right\}$, $B=\left\{b_{1}, b_{2}\right\}$ and $C=\left\{c_{1}, c_{2}\right\}$ be ordered in such a way that $\omega\left(x_{1}\right) \leq 1 / 2 \leq \omega\left(x_{2}\right)$ for $x=a, b, c$. Observe that $a_{1} b_{2}$ and $a_{1} c_{2}$ cannot both be edges, otherwise we could transfer the weight of $a_{2}$ to $a_{1}$. So $a_{1}$ is joined to one of $b_{1}, c_{1}$. Without loss of generality, suppose $a_{1} b_{1} \in E(G)$. Then $a_{1} c_{2} \in E(G)$ by condition (1), and $b_{1} c_{2} \in E(G)$ by a condition analogous to (1) on the two vertices of $B$. Thus $\left(a_{1}, b_{1}, c_{2}\right)$ forms a triangle. Now suppose some vertex in some part, say $b_{2} \in B$, is not joined to another part, say $C$. Let $N_{A}\left(b_{1}\right)$ (resp. $N_{C}\left(b_{1}\right)$ ) be the neighbour set of $b_{1}$ in $A$ (resp. $C$ ). Let $w\left(b_{1}\right)=b$, and let the total weights of vertices in $N_{A}\left(b_{1}\right)\left(\right.$ resp. $\left.N_{C}\left(b_{1}\right)\right)$ be $a$ (resp. c). Then $\alpha=b c, \beta \leq 1-a c$ (since there is no edge between $N_{A}\left(b_{1}\right)$ and $N_{C}\left(b_{1}\right)$ ) and $\gamma \leq 1-b(1-a)$ (since there is no edge between $b_{1}$ and $A \backslash N_{A}\left(b_{1}\right)$ ). Then

$$
\begin{aligned}
& c(1-b)(\alpha \beta+\gamma-1)+b(1-c)(\gamma \alpha+\beta-1) \\
\leq & c(1-b)(b c(1-a c)-b(1-a))+b(1-c)(b c(1-b+a b)-a c) \\
= & b c(1-b)(1-c)(a c-a b+b-1) \\
\leq & b c(1-b)^{2}(1-c)(a-1) \leq 0 .
\end{aligned}
$$

Thus either $\alpha \beta+\gamma-1 \leq 0$ or $\gamma \alpha+\beta-1 \leq 0$, a contradiction.

Corollary $3 t_{3}=\tau \approx 0.618$, the golden ratio.

Problem 1 Determine $t_{n}$, for $4 \leq n<\omega$.

We suspect that $t_{n}$ is strictly greater than $1 / 2$ for all $n \geq 3$. The following construction shows that $t_{4}>1 / 2$. Take a circuit $a_{1} b_{2} d_{1} a_{2} c_{1} a_{3} b_{1} d_{2} a_{1}$. Then add the edges $a_{1} c_{1}$ and $d_{1} b_{1}$. Finally add a vertex $c_{2}$ and join it to $b_{2}, a_{2}, a_{3}, d_{2}$. We assign weights to the vertices as follows: $w\left(a_{1}\right)=r_{1}, w\left(a_{2}\right)=w\left(a_{3}\right)=\left(1-r_{1}\right) / 2, w\left(b_{1}\right)=r_{2}, w\left(b_{2}\right)=1-r_{2}, w\left(c_{1}\right)=r_{3}, w\left(c_{2}\right)=1-r_{3}$, $w\left(d_{1}\right)=r_{2}$, and $w\left(d_{2}\right)=1-r_{2}$. The partition consists of $\left\{a_{1}, a_{2}, a_{3}\right\},\left\{b_{1}, b_{2}\right\},\left\{c_{1}, c_{2}\right\}$ and $\left\{d_{1}, d_{2}\right\}$. Choosing $r_{1}=0.66, r_{2}=0.3$ and $r_{3}=0.26$, the minimal edge density is 0.51 . 


\section{Locally balanced tripartite graphs.}

A multipartite graph $G$ is locally balanced if, for every $i, 1 \leq i \leq n$, each vertex $x \in X_{i}$ has the same number of neighbours in every subset $X_{j}, j \neq i$.

Theorem 4 Let $G$ be a locally balanced tripartite graph with edge densities $\alpha, \beta, \gamma$, where

$$
\max \{\alpha+\beta, \beta+\gamma, \gamma+\alpha\}>1 \text {. }
$$

Then $G$ has a triangle.

Proof. We prove the contrapositive. Suppose that $G$ has no triangle. Let $G$ have parts $A, B, C$, and consider an edge $x y$ with $x \in A$ and $y \in B$. Since $x y$ belongs to no triangle of $G$,

$$
d_{C}(x)+d_{C}(y) \leq|C|
$$

where $d_{C}(x):=\left|N_{C}(x)\right|$. Summing over all edges $x y$ with $x \in A$ and $y \in B$, we have

$$
\sum_{x y \in E \cap(A \times B)} d_{C}(x)+d_{C}(y) \leq \gamma|A||B||C|,
$$

that is,

$$
\sum_{x \in A} d_{C}(x) d_{B}(x)+\sum_{y \in B} d_{C}(y) d_{A}(y) \leq \gamma|A\|B\| C| .
$$

Because $G$ is locally balanced, $d_{B}(x)=d_{C}(x)$ for all $x \in A$, and $d_{A}(y)=d_{C}(y)$ for all $y \in B$. Making these substitutions, and applying the Cauchy-Schwartz inequality, yields

$$
\frac{\left(\sum_{x \in A} d_{C}(x)\right)^{2}}{|A|}+\frac{\left(\sum_{y \in B} d_{C}(y)\right)^{2}}{|B|} \leq \gamma|A||B||C| .
$$

Noting that

$$
\sum_{x \in A} d_{C}(x)=\beta|A||C|, \quad \text { and } \quad \sum_{y \in B} d_{C}(y)=\alpha|B||C|,
$$

we obtain

$$
\beta^{2}|A||C|+\alpha^{2}|B||C| \leq \gamma|A||B| .
$$

But $\beta|A||C|=\alpha|B||C|=\gamma|A||B|$, so $\alpha+\beta \leq 1$. Likewise, $\beta+\gamma \leq 1$ and $\gamma+\alpha \leq 1$.

Corollary 4 Let $G$ be a locally balanced tripartite graph. If all edge densities $\alpha, \beta, \gamma$ are greater than 1/2, G has a triangle.

Corollary 5 (Mantel, Turán) If a graph $G$ on $n$ vertices has more than $n^{2} / 4$ edges, it contains a triangle.

Proof. Take three copies $V_{1} V_{2}$ and $V_{3}$ of the vertex set of $G$, and add the edges $x_{i} y_{j}$ whenever $x y$ is an edge of $G$ and $i \neq j \in\{1,2,3\}$. The resulting tripartite graph is locally balanced. By Corollary 4, this graph has a triangle, which corresponds to a triangle of $G$. 


\section{Bipartite subgraphs versus triangle-free subgraphs.}

We have been investigating dense triangle-free multipartite graphs, and in particular tripartite graphs. We now consider triangle-free subgraphs of graphs in general. Denote by $f_{t}(G)$ the largest number of edges in a triangle-free subgraph of $G$. This parameter was introduced by Erdős [1], who also defined $f_{b}(G)$ to be the largest number of edges in a bipartite subgraph of $G$. Clearly $f_{t}(G) \geq f_{b}(G)$, and Turán's theorem for triangles says that equality holds when $G$ is a complete graph. V.T. Sós asked (see [1]) if this is so for every chordal graph, or perhaps even for graphs in which every odd circuit has a chord. The answer is negative.

Consider the complete graph with vertices $x_{1}, x_{2}, x_{3}, x_{4}, x_{5}$. Add five vertices $y_{1}, y_{2}, y_{3}, y_{4}, y_{5}$ and the 10-circuit $C=x_{1} y_{1} x_{2} y_{2} x_{3} y_{3} x_{4} y_{4} x_{5} y_{5} x_{1}$. The resulting graph $G$ is chordal. Now $f_{t}(G) \geq$ 15 because $C$ together with the 5 -circuit $x_{1} x_{3} x_{5} x_{2} x_{4} x_{1}$ is triangle-free, whereas $f_{b}(G) \leq 14$ since $G$ is the disjoint union of five triangles and one 5 -circuit. (In fact, both inequalities are equalities.)

Erdős[1] raised the question of giving a general condition implying that $f_{t}(G)=f_{b}(G)$. In Theorem 5, we give a sufficient condition in terms of the minimum degree.

Theorem 5 If $G$ is a graph with $n$ vertices and minimum degree at least $17 n / 20$, then $f_{t}(G)=$ $f_{b}(G)$.

Proof. Let $B$ be a largest bipartite subgraph of $G$. Erdös observed that $B$ has at least $17 n^{2} / 80$ edges, since the degree of every vertex of $B$ is at least the half of its degree in $G$. Let $H$ be a largest triangle-free subgraph in $G$. We claim that $|E(H)|=|E(B)|$. Clearly $|E(H)| \geq|E(B)| \geq 17 n^{2} / 80$. If $H$ has a vertex $x_{1}$ of degree at most $2 n / 5$, we delete it. If $H \backslash x_{1}$ has a vertex $x_{2}$ of degree at most $2(n-1) / 5$, we delete it. We continue in this way until we obtain a graph $M$ with $m$ vertices and minimum degree greater than $2 m / 5$. By a theorem of Häggkvist [3], $M$ is bipartite. The claim $|E(H)|=|E(B)|$ follows trivially if $m=n$. Now suppose $m<n$. As

$$
\frac{m^{2}}{4} \geq|E(M)| \geq|E(H)|-\frac{2}{5}\left(\left(\begin{array}{l}
n \\
2
\end{array}\right)-\left(\begin{array}{c}
m \\
2
\end{array}\right)\right) \geq \frac{17 n^{2}}{80}-\frac{n^{2}-m^{2}}{5},
$$

it follows that $m \geq n / 2$. Let $V(G) \backslash V(M)=\left\{x_{1}, x_{2}, \ldots, x_{n-m}\right\}$. Define $F=\{e \in E(G)$ : $e$ is incident to at least one $\left.x_{i}, 1 \leq i \leq n-m\right\}$. Then

$$
|F| \geq \sum_{i=1}^{n-m} d_{G}\left(x_{i}\right)-\left(\begin{array}{c}
n-m \\
2
\end{array}\right) \geq \frac{17 n(n-m)}{20}-\left(\begin{array}{c}
n-m \\
2
\end{array}\right) .
$$

Now one can construct a bipartite graph by adding vertices $x_{1}, \ldots, x_{n-m}$ together with at least half the edges in $F$ to $M$, using the Erdös observation that each $x_{i}$ is added to the partite set so that it has at least half the edges (in $G$ ) incident to the opposite partite set. So, the number of edges in the resulting bipartite graph is at least

$$
|E(H)|-\frac{2}{5}\left(\left(\begin{array}{c}
n \\
2
\end{array}\right)-\left(\begin{array}{c}
m \\
2
\end{array}\right)\right)+\frac{1}{2}|F| \geq|E(H)|+\frac{(n-m)(2 m-n)}{40} \geq|E(H)| .
$$


This implies that $|E(B)| \geq|E(H)|$ and thus $|E(B)|=|E(H)|$.

Let $c$ denote the smallest real number such that, for every sufficiently large graph with minimum degree greater than $c n$, we have $f_{t}(G)=f_{b}(G)$. Theorem 5 shows that $c \leq 0.85$. In Theorem 6 , we establish the lower bound $c \geq 27 / 40=0.675$. We need the following proposition.

Proposition 1 Let $G$ be the lexicographic product $C_{5}\left[K_{m}\right]$. Then

(i) $f_{t}(G)=5 m^{2}$.

(ii) $f_{b}(G)=17 m^{2} / 4$.

Proof. Denote by $V_{i}, 1 \leq i \leq 5$, the vertex sets of the five copies of $K_{m}$ in $G$ (in cyclic order).

(i). Let $H$ be a largest triangle-free subgraph of $G$. Consider a multi-covering of $G$ by triangles of the form $x y z x$ with $x, y \in V_{i}$ and $z \in V_{i+1}, 1 \leq i \leq 5$. Let $\left\{T_{i}: 1 \leq i \leq t\right\}$ be the set of such triangles, where $t=5 m\left(\begin{array}{c}m \\ 2\end{array}\right)$. Each edge of $G$ appears in either $m-1$ or $m$ of these triangles, according to whether it corresponds to an edge of $C_{5}$ or an edge of $K_{m}$. Therefore,

$$
\sum_{i=1}^{t}\left|E\left(H \cap T_{i}\right)\right| \geq(m-1)|E(H)|=(m-1) f_{t}(G) .
$$

On the other hand, because $H$ is triangle-free, $\left|E\left(H \cap T_{i}\right)\right| \leq 2,1 \leq i \leq t$, and so

$$
\sum_{i=1}^{t}\left|E\left(H \cap T_{i}\right)\right| \leq 2 t=5 m^{2}(m-1) .
$$

These two inequalities yield the bound $f_{t}(G) \leq 5 m^{2}$. Since the subgraph $C_{5}\left[\overline{K_{m}}\right]$ of $G$ is triangle-free and has $5 m^{2}$ edges, we conclude that $f_{t}(G)=5 m^{2}$.

(ii). Let $B(X, Y)$ be a largest bipartite subgraph of $G$. Denote by $V_{i}, 1 \leq i \leq 5$, the vertex sets of the five copies of $K_{m}$ in $G$ (in cyclic order), and set $x_{i}:=\left|X \cap V_{i}\right|, 1 \leq i \leq 5$. Then

$$
\begin{aligned}
e(B) & =\sum_{i=1}^{5} x_{i}\left(m-x_{i}\right)+\sum_{i=1}^{5} x_{i}\left(m-x_{i+1}\right)+\sum_{i=1}^{5} x_{i+1}\left(m-x_{i}\right) \\
& =3 m x-x^{2}+2 \sum_{i=1}^{5} x_{i} x_{i+2},
\end{aligned}
$$

where $x:=\sum_{i=1}^{5} x_{i}=|X|$. We first maximize $\sum_{i=1}^{5} x_{i} x_{i+2}$ for fixed $x$; this is clearly equivalent to maximizing $\sum_{i=1}^{5} x_{i} x_{i+1}$. We assume that the indices are read modulo 5 .

Claim 1 If $x_{i}>x_{i+1}$, either $x_{i+2}=0$ or $x_{i+4}=m$.

Proof. Suppose to the contrary that $x_{i+2}>0$ or $x_{i+4}<m$. Decreasing $x_{i+2}$ by 1 and increasing $x_{i+4}$ by 1 increase the number of edges by $x_{i}-x_{i+1}$, a contradiction. 
Corollary 6 1. If $x_{i}=x_{i+2}=m$, either $x_{i+1}=m$ or $x_{i+3}=x_{i+4}=m$.

2. If $x_{i}=m$ and $x_{j}<m$ for all $j \neq i$, then $x_{i+2}=x_{i+3}=0$.

3. If $x_{i}=x_{i+2}=0$, either $x_{i+1}=0$ or $x_{i+3}=x_{i+4}=0$.

4. If $x_{i}=0$ and $x_{j}>0$ for all $j \neq i$, then $x_{i+2}=x_{i+3}=m$

5. If $0<x_{i}<m, 1 \leq i \leq 5$, then $x_{i}=x_{j}, i \neq j$.

Set

$$
N_{0}:=\left|\left\{i: x_{i}=0\right\}\right|, \quad N_{m}:=\left|\left\{i: x_{i}=m\right\}\right| .
$$

Applying the above corollary, it can be shown that the maximal values of $\sum_{i=1}^{5} x_{i} x_{i+1}$ for fixed $x$ are given by the following table:

\begin{tabular}{|c|c|c|c|}
\hline$N_{m}$ & $N_{0}$ & range of $\mathrm{x}$ & $\max \sum_{i=1}^{5} x_{i} x_{i+1}$ \\
\hline 0 & 0 & $0<x<5 m$ & $x^{2} / 5$ \\
0 & 1 & none & none \\
0 & 2 & $0 \leq x \leq 2 m$ & $x^{2} / 4$ \\
0 & 3 & $0 \leq x \leq 2 m$ & $x^{2} / 4$ \\
0 & 4 & $0 \leq x \leq m$ & 0 \\
0 & 5 & $x=0$ & 0 \\
1 & & $m \leq x \leq 3 m$ & $m(x-m)$ \\
2 & & $2 m \leq x \leq 3 m$ & $m(x-m)$ \\
2 & & $x \geq 3 m$ & $m^{2}+(x-m)^{2} / 4$ \\
3 & & $x \geq 3 m$ & $m^{2}+(x-m)^{2} / 4$ \\
4 & & $x \geq 4 m$ & $m(2 x-5 m)$ \\
5 & & $x=5 m$ & $5 m^{2}$ \\
\hline
\end{tabular}

Thus,

$$
\sum_{i=1}^{5} x_{i} x_{i+1} \leq\left\{\begin{array}{rr}
x^{2} / 4, & 0 \leq x \leq 2 m \\
m(x-m), & 2 m \leq x \leq 3 m \\
m^{2}+(x-m)^{2} / 4, & 3 m \leq x \leq 5 m
\end{array}\right.
$$

and finally,

$$
3 m x-x^{2}+2 \sum_{i=1}^{5} x_{i} x_{i+1} \leq\left\{\begin{array}{rr}
4 m^{2}, & 0 \leq x \leq 2 m \\
17 m^{2} / 4, & 2 m \leq x \leq 3 m \\
4 m^{2}, & 3 m \leq x \leq 5 m
\end{array}\right.
$$

The construction of a bipartite subgraph of $G$ with $17 m^{2} / 4$ edges is based on the values $x_{1}=m, x_{2}=0, x_{3}=m, x_{4}=m / 2, x_{5}=0$.

Theorem 6 For $m \equiv 0(\bmod 16)$, there is a regular graph $H$ of degree $(27 m / 8)-3$ on $5 m$ vertices such that $f_{t}(H) \geq 5 m^{2}$ and $f_{b}(H) \leq 5 m^{2}-4 m$. 
Proof. Let $G$ be the lexicographic product $C_{5}\left[K_{m}\right]$. Then $G$ is a regular graph of degree $3 m-1$ on $5 m$ vertices such that $f_{t}(G) \geq 5 m^{2}$ and, by the above discussion, $f_{b}(G)=17 \mathrm{~m}^{2} / 4$. We form $H$ by adding $\left(3 m^{2} / 16\right)-m$ edge-disjoint 5 -circuits to $G$ in such a way that each vertex of $G$ lies in $(3 m / 16)-1$ of them. Thus $H$ is a regular graph of degree $(3 m-1)+(3 m / 8-2)=(27 m / 8)-3$ such that $f_{t}(H) \geq f_{t}(G) \geq 5 m^{2}$. Moreover,

$$
f_{b}(H) \leq f_{b}(G)+4\left(\left(3 m^{2} / 16\right)-m\right)=5 m^{2}-4 m,
$$

because no more than four of the five edges of each added 5-circuit can belong to a bipartite subgraph of $H$.

The authors thank Jozsef Balogh, Peter Keevash, and Benny Sudakov for pointing out a flaw in the original proof of Theorem 5 .

\section{References}

[1] P. Erdős, On some problems in graph theory, combinatorial analysis and combinatorial number theory. Proc. Cambridge Combinatorial Conf. (B. Bollobás, ed.), Academic Press (1984), 1-17.

[2] P. Erdős and M. Simonovits, A limit theorem in graph theory, Studia Sci. Math. Hungar. 1 (1966), 51-57.

[3] R. Häggkvist, Odd cycles of specified length in nonbipartite graphs. Graph theory (Cambridge), North-Holland Math. Stud., 62 (1981), 89-99

Adrian Bondy

Laboratoire LaPCS

UFR de Mathématiques

Université Claude Bernard Lyon 1

43 Boulevard du 11 Novembre

F-69622 Villeurbanne Cedex

France

jabondy@jonas. univ-lyon 1 .fr

Stéphan Thomassé

Laboratoire LaPCS

UFR de Mathématiques

Université Claude Bernard Lyon 1

43 Boulevard du 11 Novembre

F-69622 Villeurbanne Cedex

France

thomasse@jonas.univ-lyon1.fr

\author{
Jian Shen \\ Department of Mathematics \\ Texas State University \\ San Marcos, TX 78666 \\ USA \\ js480txstate.edu \\ Carsten Thomassen \\ Institute of Mathematics \\ Building 303 \\ DTU \\ DK-2800 Lyngby \\ Denmark
}

C.Thomassen@mat.dtu.dk 